\title{
NEUE ANFORDERUNGEN - VIELE OFFENE FRAGEN. ZU DEN VIELFÄLTIGEN ROLLEN VON REPOSITORIEN AM BEISPIEL DER UB WIEN
}

\section{von Susanne Blumesberger}

Zusammenfassnung: Der Aufbau und Betrieb von Repositorien stellt wissenschaftliche Bibliotheken vor komplexe Herausforderungen. Zahlreiche technische, juristische und ethische Fragen sind ungelöst und können nur in einer engen Zusammenarbeit mit einer Vielzahl an ExpertInnen aus unterschiedlichen Fachrichtungen beantwortet werden. Die größte Aufgabe und zugleich eine wichtige Zukunftsperspektive für Bibliotheken besteht darin, sich rasch auf ändernde technische Gegebenheiten, Publikationskulturen und wissenschaftliche Arbeitsabläufe einzustellen und den Forschenden Tools anzubieten, die nicht nur das bloße Ablegen von Daten ermöglichen sondern weit darüber hinaus den gesamten Forschungslebenszyklus optimal unterstützen.

Schlüsselwörter: Repositorien; Forschungsdaten; wissenschaftliche Bibliotheken; Forschungsdatenlebenszyklus; Datenmanagement

\section{NEW REQUIREMENTS - MANY OPEN QUESTIONS. ON THE DIVERSE ROLES OF REPOSITORIES USING THE EXAMPLE OF THE VIENNA UNIVERSITY LIBRARY}

\begin{abstract}
The construction and operation of repositories presents academic libraries with complex challenges. Numerous technical, legal and ethical questions remain unresolved and can only be answered in close cooperation with a large number of experts from different disciplines. The biggest challenge, and at the same time an important future for libraries, is to be able to adapt quickly to changing technical conditions, publication cultures and scientific workflows, and to offer researchers tools that not only allow data to be stored, but also optimally cover the entire research life cycle support.
\end{abstract}

Keywords: Repositories; research data; academic libraries; research data life cycle; data management

DOI: http://doi.org/10.31263/voebm.v71i1.2003

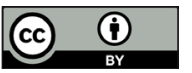

Dieses Werk ist lizenziert unter einer

Creative-Commons-Lizenz Namensnennung 4.0 International 


\section{Inhalt}

1. Einleitung

2. Repositorien - von der Datenarchivierung zum vielfältigen Instrumentarium

3. Das Chamäleon Repositorium?

4. Forschungsunterstützung morgen?

5. Die Archivierung von Forschungsdaten in der Zukunft

Bibliotheken müssen ihr Geschäftsmodell radikal ändern. Wer das nicht tut, der wird in den nächsten 20 Jahren verschwinden. ${ }^{1}$

Rafael Ball

\section{Einleitung}

In den letzten Jahren gab es einen unübersehbaren Wandel in den Bibliotheken, vor allem forschungsunterstützende Services und hier meist die langfristige Sicherung der Daten betreffend. Die Erwartungen und Anforderungen an Repositorien passten sich gemäß der veränderten Arbeitsgewohnheiten der Wissenschaftlerlnnen ebenfalls an. Eine Überlegung, die hier gestellt werden soll ist, inwieweit Repositorienbetreibende die sich ändernden Erwartungen und Forderungen berücksichtigen können und sollen. Einige Fragen, die sich in diesem Zusammenhang stellen werden: Wo braucht es technisch und nichttechnisch gesehen Kontinuität und Stabilität, wo sind ständige Anpassungen angebracht? Welche Infrastrukturen werden in Zukunft nötig sein um möglichst auf alle Bedürfnisse der Forschenden eingehen zu können? Auch auf inhaltlicher Ebene ist ein ständiger Diskurs wichtig. Die Frage des Zugangs zum Repositorium, beispielsweise die Entscheidung ob alle MitarbeiterInnen mit dem eigenen Account archivieren dürfen oder ob das Archivieren nur einer eingeschränkten Benutzergruppe möglich sein soll, muss ebenso hinterfragt und entschieden werden, wie die grundsätzliche Festlegung, wer bestimmen darf, was langfristig verfügbar gehalten werden soll? Denn davon leitet sich im Grunde genommen ab, wer heute das kulturelle Erbe von morgen definiert. Abgesehen von diesen Überlegungen stellt sich auf Datenebene stets die Frage, wie man einerseits den geforderten FAIR-Principles ${ }^{2}$ entsprechen kann, die Daten also auffindbar, zugänglich, austauchbar und wiederverwendbar hält und andererseits bewusst mit jenen Daten umgeht, die aus rechtlichen und/oder ethischen Gründen eines sehr reflektierten und vorsichtigen Umgangs bedürfen. Von diesem Spannungsfeld zwischen den Forderungen nach Open Science und Open Data auf der einen Seite und einer hohen 
Sensibilität gegenüber Daten auf der anderen Seite sind nicht nur Wissenschaftlerlnnen der unterschiedlichen Fachdisziplinen betroffen, sondern auch Bibliothekarlnnen, Archivarlnnen und Sammlungsbeauftragte, denn auch für diese Personengruppen sind institutionelle Repositorien wichtig. Bei sensiblen Daten, die evtl. eines eingeschränkten Zugangs, einer Pseudonymisierung, Anonymisierung und besonderen technischen Sicherheitsvorkehrungen bedürfen, tragen Repositorien eine hohe Verantwortung im Bereich Datenschutz und sind nicht nur in der Forschung unverzichtbar geworden, sondern übernehmen auch gesellschaftspolitische Aufgaben.

\section{Repositorien - von der Datenarchivierung zum vielfältigen Instrumen- tarium}

Zehn Jahre bedeuten in der digitalen Welt eine lange Zeitspanne. Entsprechend den technischen Gegebenheiten - Handys waren noch nicht so smart wie heute und Tablets kaum verbreitet - gestalteten sich die Arbeits- und Publikationsabläufe von Forscherlnnen noch etwas anders als im Jahr 2018. Digitalisierung und Retrodigitalisierung befanden sich im Aufschwung, man war als Forschender, vor allem aus den Geisteswissenschaften kommend, froh, schwer erreichbare Quellen plötzlich im Netz per Link rund um die Uhr auffinden und nutzen zu können. Ein Repositorium aufzubauen um als wissenschaftliche oder kulturelle Einrichtung selbst wertvolle Materialien weltweit digital anbieten zu können war mit einer gewissen Euphorie verbunden. Endlich hatte man die Infrastruktur zur Verfügung um bisher schwer zugängliche und deshalb kaum genutzte Schätze aus Bibliotheken und Archiven für alle Welt sichtbar und einfach nutzbar machen zu können. Zehn Jahre später gehen die meisten Forschenden selbstverständlich davon aus, dass sämtliche benötigte Quellen rund um die Uhr frei und ohne rechtliche Einschränkungen verfügbar im Netz sind und rasch kommentiert und mit anderen geteilt werden können.

Mit der Etablierung von Repositorien erwartete man sich auch einen tiefgreifenden Wandel im Bereich des Publikationswesens. Hat man als Wissenschaftlerln Zugang zu einem Repositorium, kann man prinzipiell ohne Verlag rasch und sicher eigene Texte samt Begleitmaterialien aller Art weltweit verbreiten - ohne Zeitverlust durch Peer-Review-Prozesse und Drucklegung. Der Wegfall qualitätssichernder Maßnahmen wird heute jedoch sehr kritisch gesehen. Den Open Access Policies folgend wird empfohlen entweder die Publikation im Rahmen des goldenen Wegs, also über 
Open Access-Zeitschriften oder im Rahmen des grünen Wegs, in Form von Zweitveröffentlichungen bereits peer-reviewter und publizierter Artikel vorzunehmen. Während in den Natur- und Sozialwissenschaften schon seit längerer Zeit auf diese Weise publiziert wird, herrschen in den Geisteswissenschaften oft noch Printmedien, vor allem Monografien und Sammelbände, vor. Erst langsam wird auch hier ein Umdenken sichtbar.

Einig sind sich die Forschenden bzgl. Repositorien auf jeden Fall in ihren Forderungen, dass das Hochladen eigener Publikationen und Daten sowie deren Beschreibung kaum Zeit in Anspruch nehmen darf und von allen Endgeräten aus jederzeit möglich sein soll. Archivierte Objekte müssen rasch und ohne weiteren Aufwand in unterschiedliche Systeme weiterverbreitet werden können. Nutzerlnnenzahlen, Downloadstatistiken für bibliometrische Auswertungen und diverse Evaluierungen müssen ebenfalls jederzeit problemlos abrufbar sein. Ausfälle des Systems - wenn auch noch so kurz - werden nicht mehr toleriert.

Abgesehen von den Wissenschaftlerlnnen, sind auch jene Personengruppen zu berücksichtigen, die das Repositorium dazu verwenden, Sammlungsbestände, Archivbestände, Nachlässe, historische Bücher und Karten digital zur Verfügung zu stellen und damit wieder die Datengrundlage für weitere Forschungen nutzbar machen. Auch für sie muss das Repositorium, wenn es wie PHAIDRA ${ }^{3}$ universell für eine Institution eingesetzt wird, Anwendungsmöglichkeiten bieten, die mit dem ressourcenaufwändigen Prozess der Digitalisierung und Beschreibung der Daten, in Einklang stehen und gerechtfertigt sind. Dazu zählen geeignete Uploadtools, ein effizientes Metadatenmanagement und diverse Visualisierungsmöglichkeiten. Repositorien werden oft auch für die Archivierung und Publikation von Hochschulschriften verwendet. Daraus entsteht ein ganzes Bündel an Handlungsfeldern rund um den Betrieb eines Repositoriums. Kenntnisse des Forschungsdatenzyklus, im Bereich Open Science, Open Data, Open Access, über Lizenzen, ethische und rechtliche Gegebenheiten, über technische Schnittstellen, Fragen der Langzeitarchivierung, Metadaten, Sammlungsrichtlinien, diverse Policies usw. werden erwartet. Darüber hinaus spielt auch das Wissen um das institutionsinterne Zusammenspiel von Forschungsservices, Drittmittelstellen und die enge Zusammenarbeit mit IT-Services eine große Rolle. Auch die von den Fördergebern teilweise bereits eingeforderten Datenmanagementpläne verlangen Beratungen hinsichtlich der optimalen Vorbereitung der Daten für die Langzeitverfügbarkeit in Repositorien.

All diese Aspekte zusammengenommen erfordern heute zugleich einen breiten Überblick über diverse Tätigkeitsfelder aber auch Spezialwissen in 
verschiedenen Bereichen, die sich zum Teil erst in den letzten Jahren entwickelt haben.

Um diese Fragen gemeinsam diskutieren zu können, wurde unter anderem das Netzwerk Repositorienmanagerlnnen ${ }^{4}$ gegründet, dem sich inzwischen zahlreiche Vertreterlnnen von Institutionen in ganz Österreich angeschlossen haben.

\section{Das Chamäleon Repositorium?}

Als 2006 an der UB Wien eine Arbeitsgruppe gegründet wurde um die Implementierung eines digitalen Repositoriums vorzubereiten, gab es kaum Erfahrungswerte, die man hreanziehen konnte. Die Repositorienlandschaft war noch sehr dünn besiedelt, das Bewusstsein für digitale Langzeitarchivierung in den meisten Wissenschaften noch kaum vorhanden. Auch die technischen Möglichkeiten waren nicht sehr breit gefächert, kein fertiges Produkt schien richtig zu passen. Die Lösung, eine Open Source Software wie Fedora, heranzuziehen und nach den Bedürfnissen einer sehr großen und heterogenen Universität zu adaptieren, lag nahe. Als im April 2007 das zunächst für drei Jahre geplante Projekt „Digital Asset Management System" startete, lag die Hauptaufgabe beim nichttechnischen Team an der Universitätsbibliothek Wien spätere NutzerInnen zu befragen, welche Aspekte dieses neuen Tools für sie wichtig seien. Gemeinsam mit Vertreterlnnen unterschiedlicher Fachrichtungen wurde ein Anforderungskatalog erstellt, der in enger Zusammenarbeit mit dem Zentralen Informatikdienst der Universität Wien abgestimmt und verfeinert wurde. Genau ein Jahr nach Projektstart ging PHAIDRA ${ }^{5}$, wie das System genannt wurde, in Betrieb. Damit war die Möglichkeit für sämtliche Mitarbeiterlnnen und Studierende gegeben, wertvolle digitale Inhalte weltweit und permanent zitierbar zur Verfügung zu stellen. Neben der ständigen Optimierung der Software und der Überarbeitung der Oberfläche, stand vor allem die Bewerbung des neuen Systems im Vordergrund. Eine Serviceseite wurde aufgebaut, die Vorteile einer langfristigen Sicherung von wertvollen Objekten angepriesen und Hilfestellung bei sämtlichen Archivierungsschritten angeboten, denn der Upload und die Beschreibung der Daten obliegen laut der Policy von PHAIDRA den Nutzerlnnen. Damit hatte die UB Wien im Bereich Repositorien eine Vorreiterrolle inne. Recht bald meldeten sich auch andere Universitäten, die auf der Suche nach Langzeitarchivierungssystemen waren und wurden Partner. 2010 schloss beispielsweise die Universität Padua einen Vertrag mit der Uni- 
versität Wien. Das dortige System wird vor allem für die Präsentation von Sammlungen verwendet. ${ }^{6}$ Inzwischen betreut die Università di Padova auch die Università Ca' Foscari und die Università luav di Venezia mit. In den letzten Jahren sind zahlreiche Universitäten und Institutionen der PHAIDRA-Community beigetreten, unter anderem die FH St. Pölten, die Kunstuniversität Linz, die Universität für angewandte Kunst in Wien, Universitäten in Serbien, Bosnien und Herzegowina und Montenegro, die vor allem Abschlussarbeiten und wissenschaftliche Beiträge archivieren. Die Landesbibliothek Vorarlberg verwendet VOLARE ${ }^{7}$ vor allem um die Kultur Vorarlbergs digital zugänglich zu machen. Fotosammlungen, Ansichtskarten, Karten und vieles mehr werden übersichtlich und in hoher Auflösung für alle zugänglich angeboten. Der FWF betreibt seine E-Book Library ${ }^{8}$, die die geförderten Open Access Publikationen enthält, auf der Basis von PHAIDRA. Jüngste PHAIDRA-Partner sind die Donau-Universität Krems, die Anton Bruckner-Privatuniversität und die Veterinärmedizinische Universität Wien. ${ }^{9}$

In den letzten Jahren konnten an zahlreichen Universitäten und anderen Forschungseinrichtungen Repositorien und diverse Infrastrukturen aufgebaut werden. Dazu hat wesentlich das dreijährige Projekt „e-Infrastructures Austria“"10, koordiniert von der UB Wien, beigetragen. 2014 vom Bundesministerium für Wissenschaft, Forschung und Wirtschaft (BMWFW) initiiert, sollte es österreichweit den koordinierten Aufbau sowie die Weiterentwicklung von Repositorieninfrastrukturen fördern. In den drei Jahren konnte viel Know-how in den Bereichen Open Access, Technik, rechtliche Fragen, Metadaten usw. aufgebaut werden. Es entstand ein umfassendes Netzwerk mit Bibliothekarlnnen, TechnikerInnen, ForscherInnen, Forschungsservices und anderen ExpertInnen sowie ein Wissenspool an dem alle partizipieren können. Das Nachfolgeprojekt „e-Infrastructures Austria Plus“ wird - koordiniert von der Universität Innsbruck - von 2017-2019 durchgeführt. Ziel ist es, eine Infrastruktur für eScience in Österreich aufzubauen. Es werden so unterschiedliche Themen wie RDM-Policies, Datenmanagementpläne, der Aufbau von institutionellen Repositorien für Forschungsdaten, Standards für Metadaten nach den FAIR Principles, Aufbau einer DOI-Infrastruktur und erste Schritte zur Implementierung von Electronic Lab Notebooks behandelt.

Durch die Einbettung der Repositorien in die bereits bestehenden Infrastrukturen der jeweiligen Institutionen und durch die Ausweitung der Aufgaben von RepositorienbetreiberInnen entstanden neue Services, die sowohl institutionell verankert werden müssen, als auch einer großen Expertise der MitarbeiterInnen bedürfen. 


\section{Forschungsunterstützung morgen?}

Die beiden HRSM-Projekte bzw. die Erfahrungen der RepositorienmanagerInnen haben gezeigt, dass das bloße Bereitstellen von Repositorien nicht ausreicht, um WissenschaftlerInnen bei ihren Forschungen bzw. Bibliothekarlnnen, ArchivarInnen und Sammlungsbeauftragte zu unterstützen. Neben Schulungen und Workshops mit den unterschiedlichen Stakeholdern müssen zusätzlich auch weitere Dienste angeboten werden. Diese können individuell sehr unterschiedlich sein. Für Forschende zählen unter anderem auch sämtliche Fragen dazu, die auch in einem Datenmanagementplan zu finden sind, z.B. nach der Möglichkeit der Zwischenspeicherung von Materialien, nach den günstigsten Formaten, nach ethischen und rechtlichen Fragen, nach Kostenmodellen, nach der dauerhaften Abrufbarkeit von komplexen Objekten und beispielsweise Datenbanken. Aber auch technische Fragen, wie zum Beispiel nach Schnittstellen zu anderen Systemen werden immer wieder gestellt. Vor allem bei Forschungsprojekten sind inhaltliche Überlegungen rund um Metadaten und Metadatenschemata wichtig.

Die Usability der Systeme hat ebenfalls einen großen Stelenwert. Dazu zählen der Prozess des raschen und sicheren Uploadens von Publikationen und Objekten und deren Verknüpfung.

Auf strategischer Ebene ist es wichtig langfristig zu planen wie sich Repositorien in schon an den Institutionen bestehenden anderen Systemen eingliedern und optimal vernetzen lassen. Dazu kommen noch Fragen nach dem Marketing, nach der Positionierung des Repositoriums in den Institutionen bis zu Überlegungen, welche Ausbildung MitarbeiterInnen benötigen, wenn sie ein Repositorium mit all den oben beschriebenen Handlungsfeldern betreuen sollen. Diese Fragen variieren von Institution zu Institution, wobei alle RepositorienmanagerInnen immer wieder mit juristischen und ethischen Fragen konfrontiert sind.

Eine der zentralen Fragen wird sein, welchen Stellenwert Repositorien in Zukunft für die Forschung haben werden, bzw. welche Funktionalitäten gebraucht werden.

\section{Die Archivierung von Forschungsdaten in der Zukunft}

Forschungsdaten haben in der wissenschaftlichen Reputationsökonomie nur einen indirekten Wert. (Fecher 2018, 213)

Im Sinne der European Open Science Cloud (EOSC) Declaration ${ }^{11}$ heißt es: 
„European science must be grounded in a common culture of data stewardship, so that research data is recognised as a significant output of research and is appropriately curated throughout and after the period conducting the research. Only a considerable cultural change will enable long-term reuse for science and for innovation of data created by research activities: no disciplines, institutions or countries must be left behind." (European Open Science Cloud Declaration, 1).

Demnach müssen Daten per default Open Access sein. Großen Wert wird auf Research Datamanagement, Data Stewardship, Datenmanagementpläne und die Einhaltung der FAIR-Principles gelegt.

Wie müssen Repositorien der Zukunft beschaffen sein um auf diese Anforderungen reagieren zu können? Aber auch weitere zukunftsweisende Überlegungen sind wichtig: Für welche Zwecke werden Repositorien zukünftig eingesetzt werden? Werden eher Fachrepositorien wichtig sein oder institutionelle Repositorien, die, wie PHAIDRA heute, offen für sämtliche Daten sind? Welche Rolle spielen Forschungsdaten in Zukunft. Werden diese wirklich wiederverwendet? Inwieweit lässt sich die Forderung nach Daten gemäß den FAIR-Principles realisieren? In vielen Forschungsbereichen, wie etwa der Medizin oder in der Ethnologie unterliegen die Forschungen juristischen und/oder ethischen Einschränkungen.

Mit dem Appell zur Offenheit wird von Seiten der Förderer und politischen Entscheidungsträger an Digitalisierung ein großes Fortschrittsversprechen geknüpft, das bislang noch nicht eingelöst wird. Am Beispiel von Forschungsdaten wird dies überraschend deutlich. (Fecher 2018, 15)

Befürworterlnnen des Data Sharing begründen dies wie folgt:

1. Synergien und Spill-Over-Effekte, also Faktoren, die Forschung effizienter, effektiver und innovativer machen.

2. Überprüfbarkeit von Ergebnissen, also deren Replizierbarkeit und Nachvollziehbarkeit.

3. Austausch und Feedback, also die Vernetzung und Kollaboration von Wissenschaftlern" (Fecher 2018, 118)

Derzeit werden nur wenige Forschungsdaten so aufbereitet und archiviert, dass sie für die Nachnutzung in Frage kommen, wie die 2015 in Österreich durchgeführte Studie Forschende und ihre Daten gezeigt hat: Zugriff auf selbst generierte Forschungsdaten für Dritte ermöglichen Forschende in der Regel nur eingeschränkt. Während etwas mehr als die Hälfte der Befragten den Zugang nur auf Anfrage ermöglicht, stellt nur jede/jeder Zehnte seine Forschungsdaten als Open Data für die Öffentlichkeit zur Verfügung; ebenso viele verweigern den Zugriff ganz. (siehe Forschende und ihre Daten, 7) 
Jene, die ihre Daten zur Verfügung stellten, nutzten dafür kaum Repositorien: Zugriff aufForschungsdaten wird von der Mehrheit der Forschenden entweder über physische Datenträger oder per E-Mail ermöglicht. Mehr als zwei Drittel der Forschenden setzen hierfür Cloud- oder Website-Anwendungen ein; Datenarchive/Repositorien werden nur von jeder/ jedem siebenten Forschenden genutzt (siehe Forschende und ihre Daten, 7)

Der Umgang mit Forschungsdaten ist von Fach zu Fach sehr unterschiedlich wie auch die Definition, die die WissenschaftlerInnen selbst für ihre Forschungsdaten wählen. In der Studie ging man von folgender Definition aus: Unter Forschungsdaten sind alle Daten zu verstehen, die im Zuge wissenschaftlicher Forschungs- und künstlerischer Schaffensprozesse entstehen (z.B. Text, Tabellen, Video, Audio, Grafik etc.) und auf deren Grundlage ihre Forschungsergebnisse und/oder Kunstwerke basieren - z.B. durch Experimente, Quellenforschungen, Messungen, Erhebungen, Digitalisate oder Entwürfe. (siehe Forschende und ihre Daten, 15)

Die Wiederverwendung der Daten variiert ebenfalls von Fach zu Fach: Ungefähr ein Drittel der Befragten ermöglicht die Nachnutzung ihrer eigenen Forschungsdaten; tendenziell häufiger geschieht dies in der Geographie, Biologie und Chemie, verhältnismäßig seltener in der Medizin, den Sozialwissenschaften und Geisteswissenschaften. (siehe Forschende und ihre Daten, 7)

Auf die Frage, warum Forschungsdaten nicht mit anderen geteilt werden, wurden unter anderem „der erhöhte Zeit- und Kostenaufwand, ein möglicher Datenmissbrauch, rechtliche Unsicherheiten, eine potenzielle Datenverfälschung, unerwünschte Kommerzialisierung sowie Erhöhung des Konkurrenzdrucks" genannt. (Forschende und ihre Daten, 7) Vor allem in der Medizin, den Sozial- und Verhaltenswissenschaften sowie den Ingenieurwissenschaften nannten die Befragten rechtliche Einschränkungen als größte Hindernisse. (siehe Forschende und ihre Daten, 7f.)

Fecher verweist in seiner Studie jedoch auch auf ein anderes Hemmnis: Im Mai 2016 veröffentlichten die Autoren Dan L. Longo und Jeffrey M. Drazen den Leitartikel „Data Sharing“ in der renommierten Fachzeitschrift New England Journal of Medicine (NEJM). In dem Artikel bezeichneten sie Wissenschaftlerlnnen, die für ihre Forschung Daten anderer Wissenschaftler verwenden, als „research parasites“. (Longo und Drazen 2016, 276)

Häufig werden auch rechtliche und ethische Bedenken genannt, wenn Daten nicht geteilt werden: Rechtliche Unsicherheiten entstehen weiterhin aufgrund der fehlenden Verständigung auf einheitliche Lizenzmodelle zur Nachnutzung von Daten. Im Bereich der Artikelpublikationen hat sich mit den Creative Commons-Lizenzen ein Quasi-Standard zur Beschreibung der Open Access-Bedingungen durchgesetzt. Vor dem Hintergrund des Aus- 
baus der europäischen Dateninfrastruktur, insbesondere mit Hinblick auf die geplante European Open Science Cloud, sind verstärkte Harmonisierungsbemühungen und die Verständigung auf Standard-Lizenzmodelle dem Austausch zuträglich. (Fecher 2018, 132)

Aber auch folgende Gründe können gegen eine Nachnutzung von Daten sprechen:

1. die Falsifizierung, also der Nachweis der Ungültigkeit einer Aussage des Primärforschers durch den Nachnutzer,

2. die nachteilige kommerzielle Nutzung, als die nachteilige oder unerwünschte Nutzung der bereitgestellten Daten durch kommerzielle Unternehmen,

3. die kompetitive Nutzung, also die Publikation von Ergebnissen auf Grundlage von bereitgestellten Daten durch konkurrierende Forscher und

4. die fehlerhafte Interpretation der bereitgestellten Daten durch andere Wissenschaftler. (Fecher 2018, 139)

Da die Publikationen für Forschende im Vordergrund stehen, weil derzeit ihre Forschungsleistung daran gemessen wird, wäre es evtl. denkbar, Forschungsdaten erst zeitverzögert einzufordern, erst nach der Veröffentlichung der Daten in Form einer Publikation. Datenzeitschriften oder Anerkennungen für viel genutzte Daten könnten weitere Anreize schaffen, wie es auch die EOSC empfiehlt: „Researchers who make research data open and FAIR for reuse and/or reuse and reproduce data should be rewarded, both in their career assessment and in the evaluation of projects (initial funding, review of performance and impact). This should go hand in hand with other career policies in universities and research institutions (appointments, promotions etc.). "12

Bzgl. der Datenarchive stellten die Befragten keine großen Ansprüche, aber „[m]ehr als die Hälfte der Forschenden erwartet die Bereitstellung von zusätzlichem qualifiziertem Personal sowie die Verabschiedung von Policies zum Umgang mit Forschungsdaten [...] Die Mehrzahl der Forschenden wünscht sich technische Infrastrukturen sowie projektspezifische Unterstützung für das Forschungsdatenmanagement. Darüber hinaus zeigt mehr als ein Drittel Interesse an Rechtsberatung, einem allgemeinen Helpdesk sowie an Schulungsangeboten." (Forschende und ihre Daten, 8)

Die Zertifizierung von Repositorien, etwa das „Data Seal of Approval“, wird ebenfalls an Bedeutung gewinnen: „Trusted research data repositories play a fundamental role in modern science. Scientist must be able to 
find, re-use, deposit and share data via trusted data repositories that implement FAIR data principles and that ensure long-term sustainability of research data across all disciplines. Data repositories must be easy to find and identify, and provide to users full transparency about their services." ${ }^{313}$ Konzepte für eine gesicherte Langzeitarchivierung der Daten, auch jener, die sehr komplex sind, sind bisher kaum umgesetzt. Ebenso gibt es in den Bereichen Ethik und Recht noch zahlreiche ungelöste Fragen. Aber auch strukturelle Überlegungen fehlen noch, zum Beispiel, wie die langfristige Sicherung von Forschungsoutput aus zeitlich befristeten Drittmittelprojekten finanziert werden kann. Unter anderem nennt Knoche im Bereich Repositorien folgende unerledigte Aufgaben auf gesamtstaatlicher Ebene in Deutschland, die auch für Österreich gelten: Die Langzeitarchivierung der digitalen Medien, die Aufgabenteilung bei den Forschungsdaten und eine Strategie zur Retrodigitalisierung. (Knoche 2018, 120f.)

Um all diese Fragen in einem größeren Zusammenhang betrachten zu können und Einzellösungen zu vermeiden, ist es heute mehr denn je wichtig, dass die Repositorienbetreiberlnnen eng mit internen und externen Serviceeinrichtungen zusammenarbeiten und auch zunehmend die Wissenschaftlerlnnen in Diskussionen miteinzubeziehen.

\author{
Mag. ${ }^{a}$ Dr. ${ }^{\text {in }}$ Susanne Blumesberger \\ ORCID: http://orcid.org/0000-0001-9018-623X \\ Universität Wien, Bibliotheks- und Archivwesen \\ E-Mail: susanne.blumesberger@univie.ac.at
}

\title{
Literatur
}

Bauer, Bruno; Ferus, Andreas; Gorraiz, Juan; Gründhammer, Veronika; Gumpenberger, Christian; Maly, Nikolaus; Mühlegger, Johannes Michael; Preza, José Luis; Sánchez Solís, Barbara; Schmidt, Nora; Steineder, Christian (2015): Forschende und ihre Daten. Ergebnisse einer österreichweiten Befragung - Report 2015. Version 1.2. DOI: http://doi. org/10.5281/zenodo.32043. Online auch unter: http://phaidra.univie. ac.at/o:407513

Bayer, Florian; Gorraiz, Juan; Gumpenberger, Christian; Mitterauer, Lukas; Reding, Steve (2017): Sichtbarkeitssteigerung in den Geistes-, Sozial- und Kulturwissenschaften (GSK). Ergebnisse einer Befragung an der Universität Wien. Version 1.0. DOI: http://doi.org/10.5281/zenodo.400965. Online auch unter: http://phaidra.univie.ac.at/o:526603 
Blumesberger, Susanne (2009): Wissen intelligent und sicher archivieren, verbreiten und nutzbar machen. Phaidra - Das innovative digitale Langzeitarchivierungssystem der Universität Wien. In: Mitteilungen der Vereinigung Österreichischer Bibliothekarinnen und Bibliothekare 62(2), S. 7-17.

Blumesberger, Susanne (2009): Das kulturelle Erbe - sicher und langfristig in Phaidra. Digitale Langzeitarchivierung an der Universität Wien. In: B.I.T. online. Zeitschrift für Bibliothek, Information und Technologie 12(3), S. 294-296. Online unter: http://www.b-i-t-online.de/archiv/2009-03/nach2.htm

Blumesberger, Susanne (2010): Sicher archivieren - grenzenlos recherchieren - intelligent nutzen. Phaidra - digitale Langzeitarchivierung an der Universität Wien. In: Bergner, Ute; Erhard Göbel (Hg.): The ne(x) t Generation. Das Angebot der Bibliotheken. 30. Österreichischer Bibliothekartag Graz 2009. Graz, Feldkirch: Wolfgang Neugebauer Verlag, S. 197-202.

Blumesberger, Susanne (2010): Phaidra. Digitale Langzeitarchivierung an der Universität Wien. In: Fennesz-Juhasz, Christiane; Gabriele Fröschl; Rainer Hubert; Gerda Lechleitner; Siegfried Steinlechner (Hg.): Digitale Verfügbarkeit von audiovisuellen Archiven im Internet-Zeitalter. Beiträge zur Tagung der Medien Archive Austria und des Phonogrammarchivs der österreichischen Akademie der Wissenschaften. Dietrich Schüller zum 70. Geburtstag. Berlin: LIT, S. 77-84.

Blumesberger, Susanne (2014): Digitale Objekte sichern, beschreiben, archivieren und rasch verbreiten. Wie das digitale Langzeitarchivierungssystem Phaidra an der Universität Wien eingesetzt werden kann. In: Missomelius, Petra; Wolfgang Sützl; Theo Hug, Petra Grell; Rudolf Kammerl (Hg.): Medien - Wissen-Bildung. Freie Bildungsmedien und Digitale Archive. Innsbruck: Innsbruck University Press 2014, S. 127141.

Blumesberger, Susanne (2015): Die Welt der Metadaten im Universum von Repositorien. In: Mitteilungen der Vereinigung Österreichischer Bibliothekarinnen und Bibliothekare 68(3/4), S. 515-528. DOI: https:// doi.org/10.31263/voebm.v68i3.1295

Blumesberger, Susanne; Alexander Zartl (2017): Umgang mit Metadaten in Repositorien. Eine österreichweite Umfrage. In: Mitteilungen der Vereinigung Österreichischer Bibliothekarinnen und Bibliothekare 70(2), S. 249-273. DOI: https://doi.org/10.31263/voebm.v70i2.1850

Budroni, Paolo (2010): Manifest zu Bildung einer Matrix [code], Sicherung und Verschleiß des Cultural Heritage in Europa. In: Mitteilungen 
der Vereinigung Österreichischer Bibliothekarinnen und Bibliothekare 63(1/2), S. 16-24.

Budroni, Paolo; Höckner, Markus (2010): Phaidra, a Repository Project of the University of Vienna; in: iPRES 2010, 7th International Conference on Preservation of Digital Objects, Vienna. Online unter: http://phaidra.univie.ac.at/o:245909

Fecher, Benedikt (2018): Eine Reputationsökonomie. Der Wert der Daten in der akademischen Forschung. Wiesbaden: Springer VS. DOI: http:// doi.org/10.1007/978-3-658-20895-0

Knoche, Michael (2018): Die Idee der Bibliothek und ihre Zukunft. Göttingen: Wallstein Verlag.

Longo, Dan L.; Drazen, Jeffrey M. (2016). Data Sharing. New England Journal of Medicine 374(3), p. 276-277. DOI: http://doi.org/10.1056/ NEJMe1516564

1 Bibliotheken: Weg damit! NZZ am Sonntag, 7.2.2016. https://nzzas. nzz.ch/hintergrund/bibliotheken-und-buecher-weg-damit-meint-rafael-ball-Id.147683

2 https://www.force11.org/group/fairgroup/fairprinciples

3 https://phaidra.univie.ac.at/

4 https://ubifo.at/images/repmannet.pdf

5 Permanent Hosting, Archiving and Indexing of Digital Resources and Assets.

6 https://phaidra.cab.unipd.it

7 Das Vorarlberger Landesrepositorium basiert auf Phaidra. https://pid. volare.vorarlberg.at

8 https://e-book.fwf.ac.at

9 Eine Übersicht über die derzeitigen PHAIDRA-Partner befindet sich unter: https://www.phaidra.org/community/phaidra-partners/

10 http://e-infrastructures.univie.ac.at/home/

11 EOSC Declaration. https://ec.europa.eu/research/openscience/pdf/ eosc_declaration.pdf\#view=fit\&pagemode=none

12 EOSC Declaration. https://ec.europa.eu/research/openscience/pdf/ eosc_declaration.pdf\#view=fit\&pagemode=none

13 EOSC Declaration, S. 2. 